SUPPORTING INFORMATION

\title{
Trap-Assisted Transport and Non-Uniform Charge Distribution in Sulphur-Rich PbS Colloidal Quantum Dot-based Solar Cells with Selective Contacts
}

\author{
Victor Malgras ${ }^{1,2}$, Guanran Zhang ${ }^{3}$, Andrew Nattestad ${ }^{3}$, Tracey Clarke ${ }^{3}$, Attila Mozer ${ }^{3}$, \\ Yusuke Yamauchi², Jung Ho Kim ${ }^{* 1}$
}

1. Institute for Superconducting and Electronic Materials (ISEM), Australian Institute for Innovative Materials, University of Wollongong, North Wollongong, NSW 2500, Australia

2. World Premier International (WPI) Research Center for Materials Nanoarchitectonics (MANA), National Institute for Materials Science (NIMS), 1-1 Namiki, Tsukuba, Ibaraki 305-0044 (Japan)

3. Intelligent Polymer Research Institute (IPRI), ARC Centre of Excellence for Electromaterials Science, University of Wollongong, North Wollongong, NSW 2500, Australia

Corresponding authors:

Yusuke Yamauchi: $\underline{\text { Yamauchi.Yusuke@nims.go.jp }}$

Jung Ho Kim: jhk@uow.edu.au 


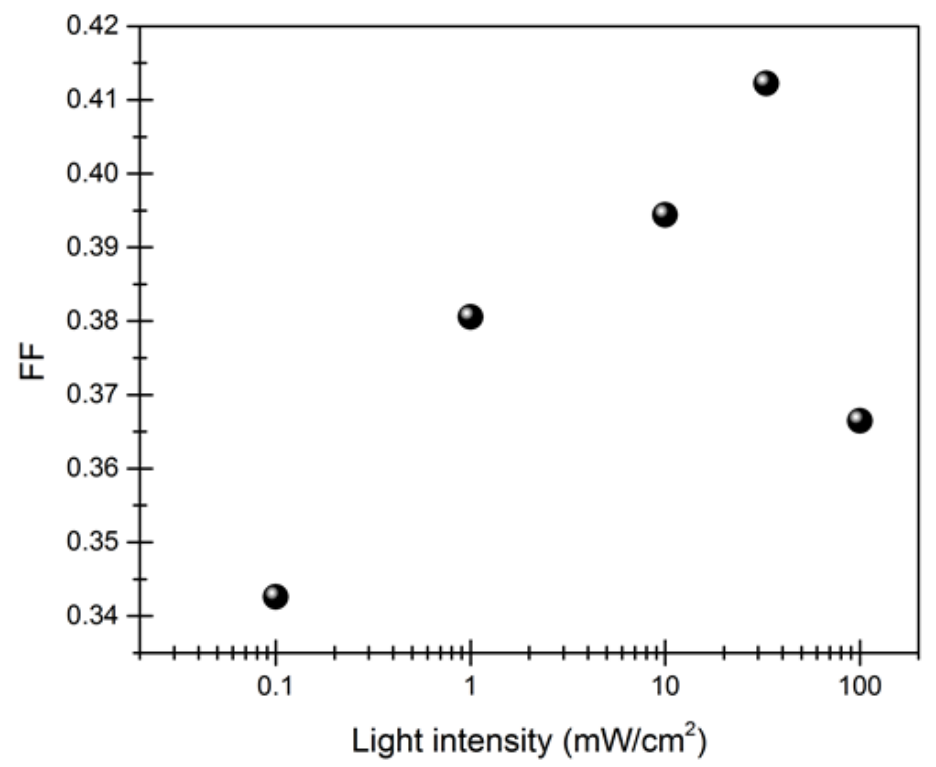

Figure S1. Fill factor (FF) as a function of the light intensity. 


\section{Fitting of dispersive exponential}

The fitting functions are:

- Geminate: $n(t)=n_{0} e^{-\left(\frac{t}{\tau}\right)^{\alpha}}$

- Non-geminate: $n(t)=\frac{n_{0}}{1+n_{0}(\beta t)^{\alpha}}$

where $n_{0}$ is the initial concentration of free photogenerated charges and $\tau, \beta$, and $\alpha$ are the geminate recombination characteristic time, the non-geminate recombination coefficient and the dispersive factor. These two functions are obtained from solving the continuity reaction: $\frac{d n}{d t}=\frac{d p}{d t}=-\beta n(t) p(t)$. In the case of geminate recombination, the equation can be simplified as the density of one charge carrier can be considered constant (e.g., $n(t)=n$ and $\beta n=\frac{1}{\tau}$ ), thus leading to a solution following a monoexponential decay. Typical non-geminate, assuming charge quasi-neutrality $(n(t) \approx p(t))$, results in a hyperbolic solution. The dispersive factor arises from taking into account the dispersive media where mobile charges have a high probability of encountering deep traps with a slow release time which compete with other characteristic transport times (diffusion, injection/transfer). For a non-dispersive random walk, the number of sites visited by the mobile charges is linear $(S(t) \propto t)$. On the other, considering a dispersion in the spatial separation between the localized states and a dispersion in the potential barriers between these sites, Scher and Montroll demonstrated that the waiting time distribution function could be well-represented by a power law $S(t) \propto t^{\alpha}$ where $\alpha=T / T_{0}$ and $T_{0}$ is the characteristic temperature of the distribution. ${ }^{1,2}$ In the limit where $T_{0}=T$ (or $\alpha=1$ ), the distribution is inexistent and the solution is non-dispersive.

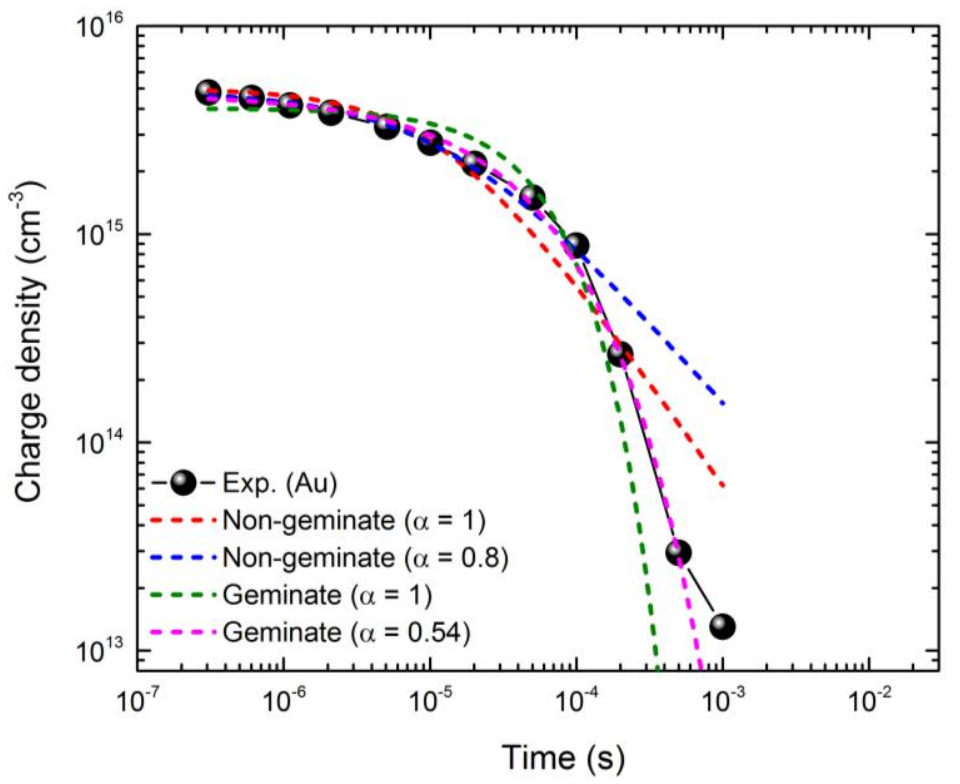

Figure S2. Experimental time-resolved charge density decay of the pixel without selective contact along with various fitting functions. 

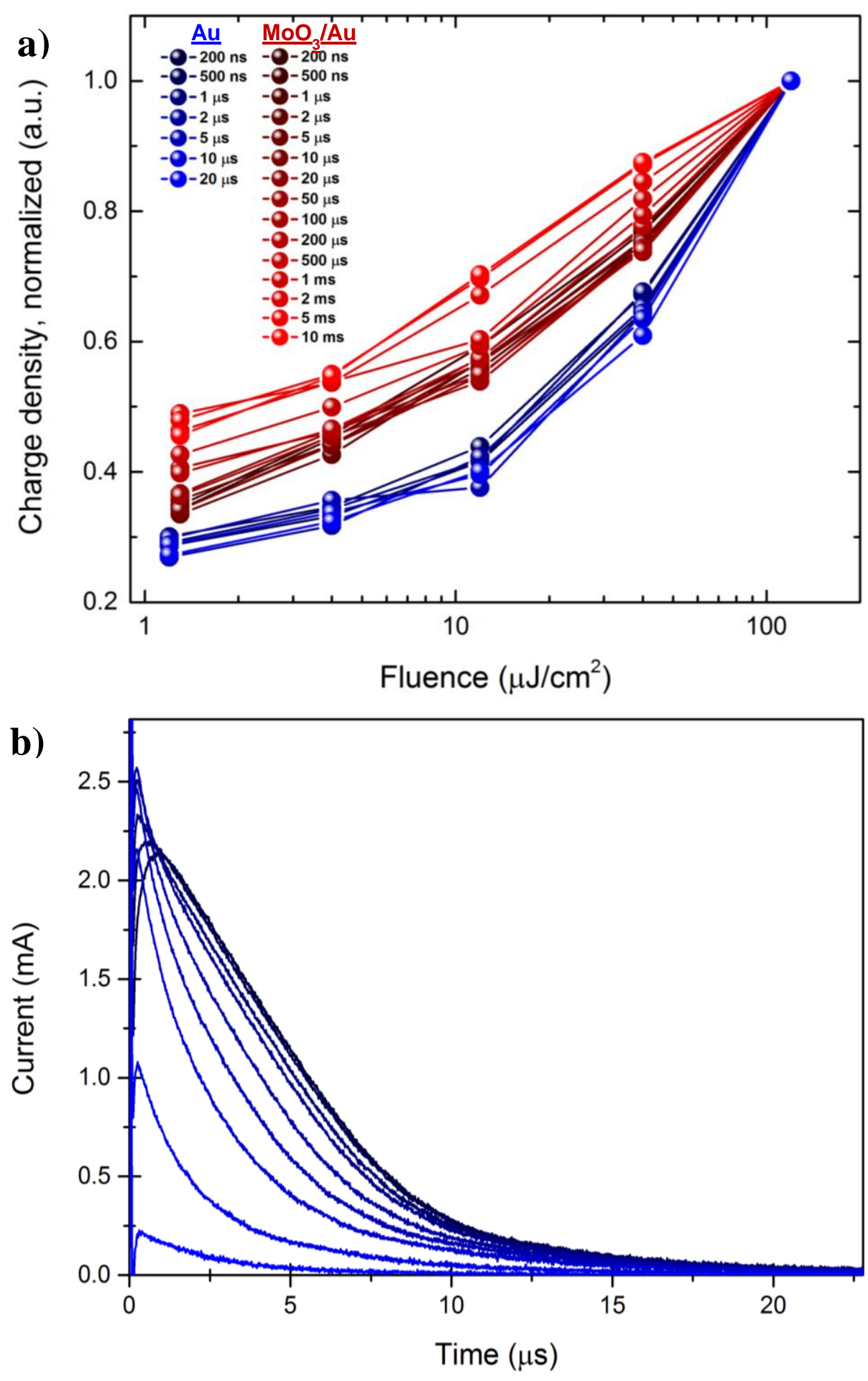

Figure S3. a) Comparison between normalized charge density in function of the light intensity, highlighting the fact that the decays are similar up to $20 \mu \mathrm{s}$. b) Current extraction curves for the device without selective contact under $40 \mu \mathrm{J} / \mathrm{cm}^{2}$ at various delay time (from $200 \mathrm{~ns}$ to $100 \mu \mathrm{s}$ ) highlighting the linear nature of the decay at fast delays. 


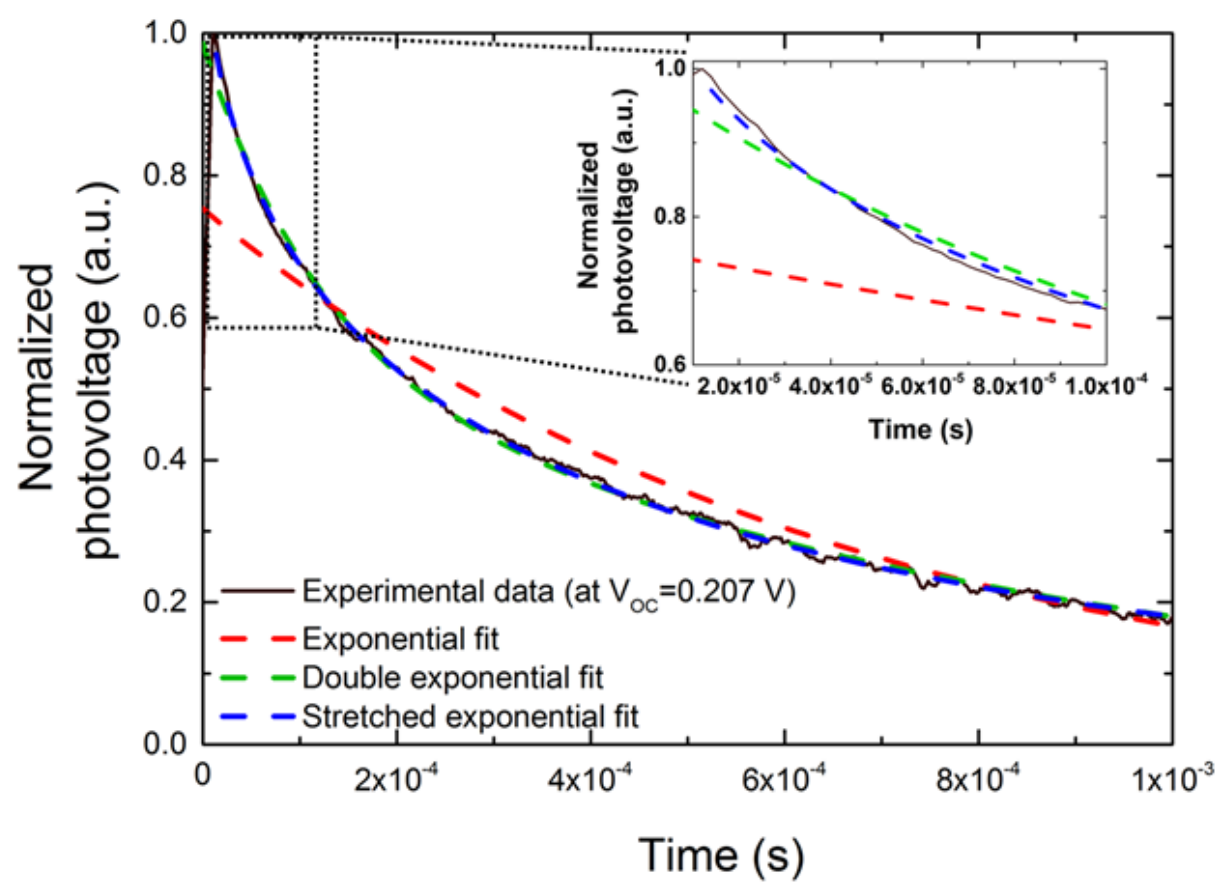

Figure S4. Typical TPV signal fitted with various exponential functions. Inset: high resolution of the early stage of the decay, highlighting the difference between the double exponential and the stretched exponential.

\section{References}

S1. Scher H.; Montroll E. W. Anomalous transit-time dispersion in amorphous solids. Phys. Rev. B 1975, 12, 2455-2477.

S2. Nelson J.; Chandler R. E. Random walk models of charge transfer and transport in dye sensitized systems. Coord. Chem. Rev. 2004, 248, 1181-1194. 